\title{
Experimental investigation of added mass effects on a Francis turbine runner in still water
}

\author{
C.G. Rodriguez ${ }^{\mathrm{a}}$, E. Egusquiza ${ }^{\mathrm{a}, *}$, X. Escaler ${ }^{\mathrm{a}}$, Q.W. Liang ${ }^{\mathrm{a}}$, F. Avellan ${ }^{\mathrm{b}}$ \\ ${ }^{a}$ Center of Industrial Diagnostics and Fluid Dynamics (CDIF), Technical University of Catalonia, Barcelona, Spain \\ ${ }^{\mathrm{b}}$ Laboratory for Hydraulic Machines (LMH), Swiss Federal Institute of Technology, Lausanne, Switzerland
}

Received 8 April 2005; accepted 1 April 2006

\begin{abstract}
The mechanical design of hydraulic turbines is conditioned by the dynamic response of the runner that is usually estimated by a computational model. Nevertheless, the runner has complex boundary conditions that are difficult to include in the computational model. One of these boundary conditions is the water in which the runner is submerged. The effect of the added mass and damping of water can modify considerably the natural frequencies of the runner. An experimental investigation in a reduced scale model of a turbine runner, using modal analysis, was carried out. Several impact tests with the runner freely suspended in air and in water were done. The response was measured with accelerometers located in different positions of the runner. From the modal analysis, the natural frequencies, damping ratios, and mode-shapes were determined. The same mode-shapes obtained in air were obtained in water but with lower natural frequencies and higher damping ratios in water. The difference in the natural frequencies is shown to be dependant basically on the added mass effect of the water and not on its added damping. This difference also depends on the geometry of the mode, presenting different values for different mode-shapes. Using nondimensional values, the reduction in the natural frequencies can be extrapolated to other Francis runners presenting similar geometrical characteristics.
\end{abstract}

(C) 2006 Elsevier Ltd. All rights reserved.

Keywords: Added mass; Natural frequencies; Turbine runner; Modal analysis

\section{Introduction}

There is a constant trend to increase the power concentration in hydraulic turbines either for new power plants or during the upgrading of existing ones. As a consequence, heads and fluid velocities are higher and the hydraulic excitation forces on the turbine runners increase. Furthermore, the operation range of hydraulic turbines is widened in order to satisfy the end-users' demand of larger regulation capacity. This operation at extreme off-design conditions leads to even larger forces.

Such forces acting alone or combined with a reduced ratio thickness/weight in runners produce high vibration levels that can cause fatigue damage [e.g., see Egusquiza et al. (2002), Fisher et al. (2002), Millán et al. (2005), Cao and Chen (2002)]. To avoid this situation it is necessary to undertake an exhaustive analysis of the dynamic behaviour of the

\footnotetext{
*Corresponding author. Tel.: + 34934016714 ; fax: + 34934015812.
}

E-mail address: egusquiza@mf.upc.edu (E. Egusquiza). 
runner. This analysis has to include the characteristics of the excitation forces and of the runner response to avoid resonances. In this paper, the response of a Francis turbine runner is analysed.

The runner response can be estimated using FEM; nevertheless, several uncertainties exist when implementing the model due to its complex geometry and boundary constrains. The boundary constrains of the runner are given by the connecting structure (rotor) and the surrounding water.

On the one hand, the runner is a component of the whole hydraulic turbine rotor which is composed by the runner, the shaft and the electrical generator. This system has its own lateral, axial and torsional natural frequencies. An analysis of the whole rotor enters into the field of rotordynamics which is especially important in machines that pass through critical velocities during the start up. However, hydraulic turbines have rigid shafts and the first natural frequency of the rotor is beyond the rotating frequency. In fact, the runner and the generator have also some natural frequencies that can be determined separately of the rest of the rotor system. Moreover, the runner is rigidly connected to the shaft of the rotor through the runner crown. At this position the main mode-shapes of the runner have low deformation. This is the reason why the attachment of the runner to the shaft will not change significantly its natural frequencies when isolated.

On the other hand, the runner is submerged in water and the effect of the added mass on the runner response can be important. The quantification of this effect is one of the main doubts when determining the runner response. It must be noted at this point that the added mass may only affect the rotor lateral frequencies, because it increases the effective mass of the runner.

Until now, there are some works presenting results of modal analysis in hydraulic runners but with measurements carried out in air (Albijanic et al., 1990). A few publications give some general data (Tanaka, 1990) but there are no detailed publications indicating the influence of the water on the modal characteristics of hydraulic runners. Other publications (Dubas and Schuch, 1987; Du et al., 1998; Xiao et al., 2001; Cao and Chen, 2002) present only results of numerical simulations without comparison with experiments.

The added mass effects due to water can also be modified by nearby structures. In an actual turbine, although there are no structures close to the blades, the casing is close to the runner around the band where the seals are located. This proximity will modify the added mass effects in the runner vibration modes with deflection against the nearby structure. So, the importance of the effect will depend on the band and seal dimensions; the longer the seals the higher the effect. In our case, the Francis runner tested is a low head runner (small pressure difference) and in this case the seals are small.

For all the reasons exposed before, the investigation of the added mass effects on a Francis turbine runner is based on an experimental modal analysis of a runner as a free body in air and in still water. Such conditions appear to be appropriate to approximately simulate the main mode-shapes of interest in an actual Francis turbine runner under operation. These modes correspond to the most susceptible ones to suffer from fatigue failure, which are the modes with high deformation in the blades.

\subsection{Added mass and damping effects of surrounding fluid}

The general equation that describes the coupling between the fluid and the structure is especially complex when the response of the structure modifies significantly the flow field leading into large vibration amplitudes. When the response of the structure does not affect the flow field, the fluid/structure interaction affects only the structural terms of the equation of motion, modifying the mass, damping and stiffness. Runners are stiff structures with streamlined blades and in normal operating conditions the effects of the response of the structure on the exciting fluid force are negligible. In this case, the flowing fluid will not significantly change the values of the natural frequencies obtained with the runner in still water (Naudascher and Rockwell, 1994; Abramson, 1969; Brennen, 1994). In view of this, the following equation is suitable to describe the change in the natural frequencies of the runner that is the objective of this study:

$$
\left(M_{1}+M_{a, 1}\right) \ddot{x}+\left(C_{1}+C_{a, 1}\right) \dot{x}+\left(k_{1}+k_{a, 1}\right) x=F(t) ;
$$

Eq. (1) represents a single degree of freedom (dof) of mass $M_{1}$, damping $C_{1}$, and stiffness $k_{1}$, with added mass $M_{a, 1}$, added damping $C_{a, 1}$, and added stiffness $k_{a, 1}$. These added coefficients are a consequence of being submerged in a still fluid.

The added mass is a portion of the surrounding fluid that is accelerated as though it were rigidly attached to the structure. This added mass effect is important when the density of the fluid is in the order of the density of the structure. For that reason, if the fluid is air, the added mass effects are negligible and the values of $M_{a, 1}, C_{a, 1}$ and $k_{a, 1}$ can be disregarded. If the fluid is water, the added mass effects cannot be neglected and the natural frequencies of the structure can be considerably altered. 
In general, the added mass is a function of the body geometry, mode-shape, submergence, nearby structures, and vibration amplitude. If the body vibrates in a flowing fluid, then the added mass may also be a function of the flow conditions. For a determined geometry and mode-shape, the higher influence is exerted by the submergence (or the proximity of a free surface) and by the nearby structures. Vibration amplitudes can affect the added mass if they are large enough to separate the fluid from the structure. In that case the motion-induced force will change from an inertia added mass-like force to a damping-like force. Experiments in cylinders (Sarpkaya, 1975; Keim, 1956) show that separation appears when vibration amplitudes are larger that 0.2 times the cylinder diameter $\left(x_{0} / d=0.2\right)$. In the tests shown later, vibration amplitudes are small and no separation is observed.

The added damping incorporated by a fluid can be important, especially when vibration amplitudes are large. The added stiffness can be important in some cases like in floating bodies.

\subsection{System parameter determination}

Some values are defined in order to represent the solution of a single dof system with mass $M$, damping $C$ and stiffness $k$. These values are the damping ratio $\zeta$, the natural frequency $\omega_{n}$, and the damped natural frequency $\omega_{d}$ :

$$
\begin{aligned}
& \zeta=\frac{C}{2 \sqrt{k M}}, \\
& \omega_{n}=\sqrt{\frac{k}{M}}, \\
& \omega_{d}=\omega_{n} \sqrt{1-\zeta^{2}} .
\end{aligned}
$$

Using these values, the solution of the single dof system can be written as $x=X \sin (\omega t-\phi)$, where $X$ is the maximum value of $x$ given by

$$
X=\frac{F / k}{\sqrt{\left[1-\left(\frac{\omega}{\omega_{n}}\right)^{2}\right]^{2}+\left[2 \zeta\left(\frac{\omega}{\omega_{n}}\right)\right]^{2}}} .
$$

The value of $X / F$ can be determined from the frequency response function (FRF) that is obtained with experimental modal analysis. All the system parameters of the single dof system can be obtained from the FRF.

The damped natural frequency $\omega_{d}$ is found at maximum FRF amplitude and for low damping ratios it can be assumed that the maximum amplitude is reached when $\omega=\omega_{n}=\omega_{d}$. The damping ratios found in this study do not exceed 0.01 . This means a difference of less than $0.005 \%$ between $\omega_{n}$ and $\omega_{d}$. In the range of frequency analysed, this difference is less than $0.5 \mathrm{~Hz}$. With this assumption, the damping ratio $\zeta$ can be determined using Eq. (6) with the frequencies $\omega_{A}$ and $\omega_{B}$, whose amplitudes correspond to half the power of the FRF at $\omega=\omega_{d}$ that equals to $(2 \zeta k)^{-1}$ (Ewins, 1984):

$$
\zeta \cong \frac{\omega_{B}-\omega_{A}}{2 \omega_{d}}
$$

Using the following equation, it is possible to determine $k$ from the values of $\zeta$ and the FRF amplitude at $\omega_{d}$ :

$$
k=\frac{1}{2 \zeta\left(\frac{X}{F}\right)_{\omega=\omega_{d}}} .
$$

It can be noticed that this procedure to determine $k$ is equivalent to using the fact that $(X / F)_{\omega=0}=1 / k$. After determining $\omega_{d}$ and $\zeta$, the mass and damping of the system can be determined using Eqs. (2)-(4) and (7):

$$
M=\frac{1-\zeta^{2}}{2 \omega_{d}^{2} \zeta\left(\frac{X}{F}\right)_{\omega=\omega_{d}}},
$$




$$
C=\frac{\sqrt{1-\zeta^{2}}}{\omega_{d}\left(\frac{X}{F}\right)_{\omega=\omega_{d}}} .
$$

Now, let us consider a multiple dof system. The FRF at any point $p$ due to a force at point $q$ for an $N$ dof system can be written as follows:

$$
\frac{X_{p}}{F_{q}}=\sum_{i=1}^{N}\left(\frac{X_{p}}{F_{q}}\right)_{i} \sin \left(\omega t-\phi_{p q, i}\right)
$$

where

$$
\left(\frac{X_{p}}{F_{q}}\right)_{i}=\frac{\alpha_{p q, i} \frac{1}{k_{i}}}{\sqrt{\left[1-\left(\frac{\omega}{\omega_{n, i}}\right)^{2}\right]^{2}+\left[2 \zeta_{i}\left(\frac{\omega}{\omega_{n, i}}\right)\right]^{2}}} .
$$

In Eqs. (10) and (11), the subscript $i$ indicates the corresponding value at the $i$ th natural frequency. It can be observed that the main difference with the response in a single dof system is the weighting value $\alpha_{p q, i}$. This value corresponds to the normalized mode-shape of the corresponding $\omega_{n, i}$. Then, the value of $\alpha_{p q, i}$ goes from -1 to 1 . If $\alpha_{p q, i}$ is zero, the point $p$ and/or $q$ is a node of the mode-shape.

Two different methods are used to extract the modal values from the FRF of the multiple dof system. In the first method it is assumed that, in the vicinity of a resonance, the response of the system is dominated by the mode at resonance. Then the only important term in Eq. (10) is due to $\omega=\omega_{n, i}$, and the resulting equation can be solved for $k_{i}$ :

$$
k_{i}=\frac{\alpha_{p q, i}}{2 \zeta_{i}\left(\frac{X_{p}}{F_{q}}\right)_{\omega=\omega_{d, i}} .}
$$

This procedure is equivalent to simplifying the model to a single dof system and it is not adequate if the modes are coupled. Coupling is found in structures where the natural frequencies are close or where the damping ratios are high. Since the damping ratios in this study are low, then the only possible coupling is due to two close natural frequencies. In this study, the difference in the closest pair of natural frequencies corresponds to $3.0 \mathrm{~Hz}$, that is 6 times the resolution in frequency of the measured data.

To avoid any possibility of coupling in the system parameter determination, a second method which considers multiple dof is used: the complex exponential (CE) method (Maia and Montalvão e Silva, 1997). Unlike other multiple dof methods, the CE has the advantage that it is not dependant on initial estimates of the system parameters. This method uses the values of the FRF at all the frequencies to solve a matrix system that is built under the assumption of different quantity of modes. It gives as many solutions as the assumed number of modes. When the number of modes assumed is less than the real one, the CE method gives solutions that clearly do not correspond to the measured data. When the amount of modes gets closer to the real number of modes, the values of natural frequencies begin to coincide with the peaks in the measured data. When the number of assumed modes is beyond the real number of modes, this method gives more solutions that can be easily separated from the real ones. Moreover, in this study, the real number of modes can be determined by the inspection of the experimental data. The solutions obtained are replaced in Eq. (10) and the values of $k_{i}$ are solved. After comparing the solutions from the two different methods, the values of damping ratios, damped natural frequencies and stiffness are determined.

Finally, the mass $M_{i}$, and the damping $C_{i}$, corresponding to each $i$ th mode are determined:

$$
\begin{gathered}
M_{i}=\frac{\alpha_{p q, i}\left(1-\zeta_{i}^{2}\right)}{2 \omega_{d, i}^{2} \zeta_{i}\left(\frac{X_{p}}{F_{q}}\right)_{\omega=\omega_{d, i}}}, \\
C_{i}=\frac{\alpha_{p q, i} \sqrt{1-\zeta_{i}^{2}}}{\omega_{d, i}\left(\frac{X_{p}}{F_{q}}\right)_{\omega=\omega_{d, i}}} .
\end{gathered}
$$


From Eqs. (12)-(14), it is possible to know the values of mass, damping, and stiffness for the system in air and in water. Even using the $\mathrm{CE}$ method, the damping ratio is hard to obtain with high accuracy and, for example, no significant difference can be observed in the FRF if the adjusted mode has a damping ratio of 0.0010 or 0.0012 . Such difference in the damping ratio can produce an error of $25 \%$ in the value of $k$.

\subsection{Determination of the added mass value}

The added effects of the fluid can be separated from the structural parameters using the values of modal mass, modal damping, and modal stiffness obtained for the system as a free body (no added effects) and for the system submerged in fluid. Comparing the equations for the system in air and in water, the following equations are obtained:

$$
\begin{aligned}
& \left(1+\frac{k_{a, i}}{k_{i}}\right)\left(1+\frac{M_{a, i}}{M_{i}}\right)^{-1}=\frac{1-\zeta^{2}}{1-\zeta_{W, i}^{2}}\left(\frac{\omega_{d, W, i}}{\omega_{d, i}}\right)^{2}, \\
& \left(1+\frac{C_{a, i}}{C_{i}}\right)^{2}\left(1+\frac{k_{a, i}}{k_{i}}\right)^{-1}\left(1+\frac{M_{a, i}}{M_{i}}\right)^{-1}=\frac{\zeta_{W, i}^{2}}{\zeta_{i}^{2}},
\end{aligned}
$$

where the values in water are labelled with subscript $W$.

The water around an entirely submerged structure does not present opposition to any constant displacement. Therefore, the stiffness effect of the water around the structure is negligible. That means that $k_{a}=0$ and the system in air has the same stiffness as the system in water. Taking this into account, Eqs. (15) and (16) can be solved for $M_{a, i} / M_{i}$ :

$$
\frac{M_{a, i}}{M_{i}}=\frac{1-\zeta_{W, i}^{2}}{1-\zeta^{2}}\left(\frac{\omega_{d, i}}{\omega_{d, W, i}}\right)^{2}-1
$$

The value of $M_{a, i} / M_{i}$ is not sensitive to the errors in the determination of the damping and it is sensitive to the value of $\omega_{d}$ that mainly depends on the frequency resolution. In this study, the frequency resolution is $0.5 \mathrm{~Hz}$ that, for the frequencies involved in this study (over $250 \mathrm{~Hz}$ ), represents less than a $0.2 \%$. Eq. (17) gives an estimation of the influence of the water in the mass of the system. A negative value means that the water subtracts mass to the system, a zero value means that the water effect is negligible and a positive value means that the water adds mass to the system.

\subsection{Nondimensional quantification of the added effect}

Making some assumptions, it is possible to generalize the values of modal added mass $M_{a, i}$ and of modal mass $M_{i}$ that are only valid for the runner tested. It is supposed that the value of $M_{a, i} / M_{i}$ only depends on the geometry of the mode, on the density of the structure $\rho_{S}$, and on the density of water $\rho_{W}$, an assumption that has been made by several authors (Blevins, 1995; Liang et al., 2001). That means that is assumed that

$$
\gamma_{i}=\frac{M_{a, i}}{\rho_{W}} \frac{\rho_{S}}{M_{i}}=\text { constant. }
$$

The added mass coefficient, $\gamma_{i}$, only depends on the geometry of the structure and is valid for any runner with similar geometry to the runner tested. It does not depend on the values of $M, C$, and $k$ determined from the FRFs and, because of the way it is calculated (Eq. (17) and the densities of the material and water), is not sensitive to the uncertainties in the calculation of the damping ratios.

If the effect of the damping in the value of frequency at maximum amplitude is neglected $(0.005 \%$ of difference for the range of damping ratios in this study), the change in the natural frequencies from air to water can be determined using the value of $\gamma_{i}$ :

$$
\omega_{n, i, W}=\frac{\omega_{n, i, A}}{\sqrt{1+\frac{M_{a, i}}{M_{i}}}}=\frac{\omega_{n, i, A}}{\sqrt{1+\frac{\rho_{W}}{\rho_{S}}} \gamma_{i}} .
$$

It must be kept in mind that these results are only valid in runners with a similar geometry, geometry that in hydraulic runners is dependant on its specific speed. 


\section{Preliminary considerations and experimental setup}

\subsection{Tested runner characteristics}

The shape of a turbine runner depends on the design requirements of net head $H$ in $\mathrm{m}$, flow rate $Q$ in $\mathrm{m}^{3} / \mathrm{s}$, and rotating speed $\Omega$ in $\mathrm{rad} / \mathrm{s}$. From dimensional analysis techniques all these characteristics are included in the specific speed $v$ defined by

$$
v=\frac{\Omega(Q / \pi)^{1 / 2}}{(2 E)^{3 / 4}},
$$

where $E$ is the specific energy given by $g H$. The most common turbine runners are of Francis type. The model runner tested is shown in Fig. 1. It is a replica at a reduced scale of 1:10 of a Francis runner with a specific speed $v$ of 0.56 , a diameter in the band of $4.09 \mathrm{~m}$, and 17 blades. The prototype runner is made of steel with a density of $7800 \mathrm{~kg} / \mathrm{m}^{3}$ and it weighs $520 \mathrm{~kg}$. The connections of the blades to the band and crown are welded. The model runner is made of bronze with a density of $8300 \mathrm{~kg} / \mathrm{m}^{3}$ and it weighs $55 \mathrm{~kg}$. The connections of the blades to the band and crown are bolted. This runner model was constructed following the International Electrotechnical Commission (IEC) Standards for international test acceptance and used in a real model scale test.

Three main parts can be observed in its construction: the crown, the blades and the band. The crown is the part that is attached to the shaft and holds one end of the blades. The blades are located between the crown and the band which holds the other end of the blades.

\subsection{Instrumentation}

To determine the runner system parameters, a series of impact tests were carried out. An impact hammer especially designed for experimental modal analysis in laboratory (model Kistler 9722) was used to excite the runner. To measure the vibratory response a small piezoelectric accelerometer (model Kistler 8704B100) was used. The signals of impact and vibratory response are conditioned with a Brüel \& Kjaer unit and recorded with a SONY DAT tape. After that, the signals are digitalized with an HP Dynamic Signal Analyser and saved in a computer for further processing.

\subsection{Test rig}

In order to get the runner system parameters as a free body with no constrains, the runner is suspended with a flexible string as is shown in Fig. 2. For the tests in water, the runner is introduced into a squared tank full of water. The natural frequencies of the supporting systems are low in comparison with the natural frequencies of the runner. The natural frequency related to the support in the vertical direction is below $10 \mathrm{~Hz}$ and the one related to the support in horizontal direction is about $3 \mathrm{~Hz}$. The first natural frequency of the runner is found to be 30 times the highest natural frequency of the support. Therefore, the effect of the support natural frequencies in the response of the runner can be neglected.

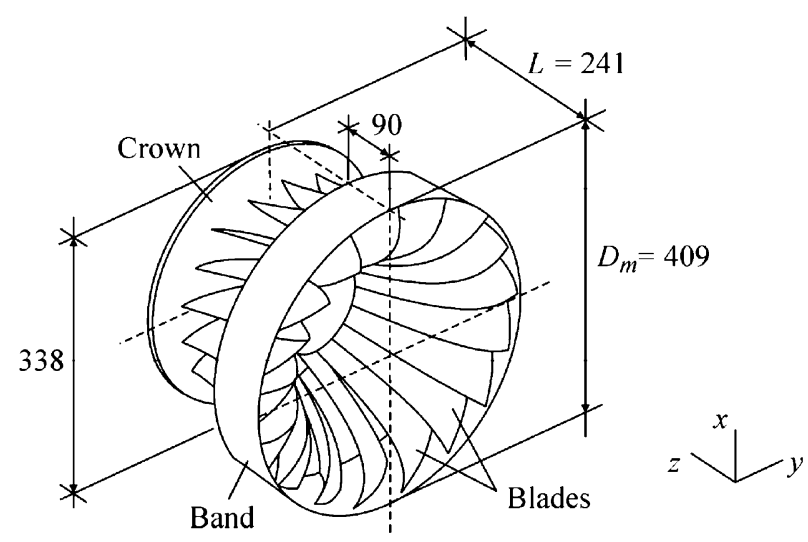

Fig. 1. Runner model and its main dimensions in $\mathrm{mm}$. 

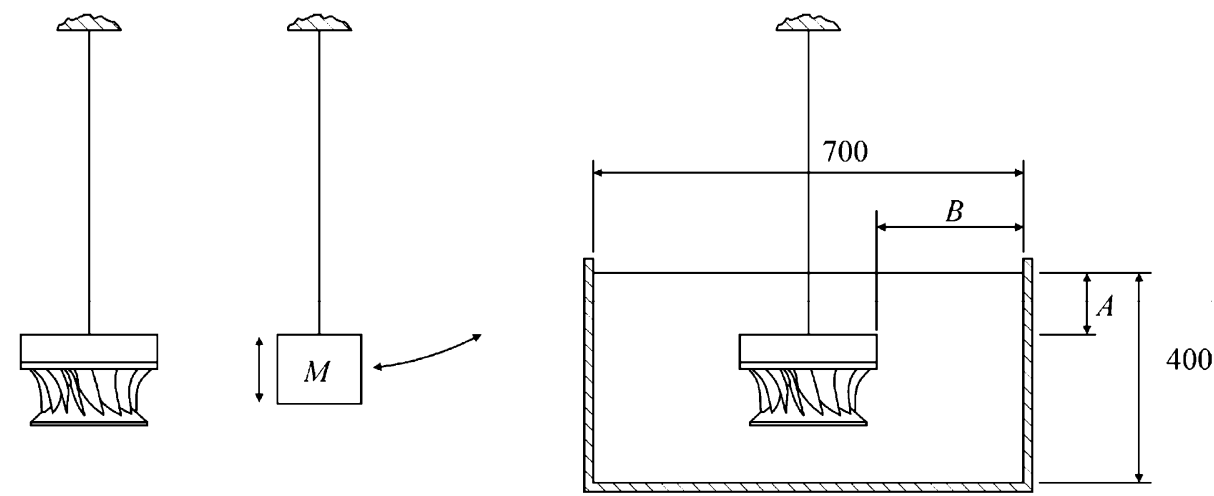

Fig. 2. Schematic of the test rig with the runner in air (left) and submerged inside water (right), all the dimensions in mm.

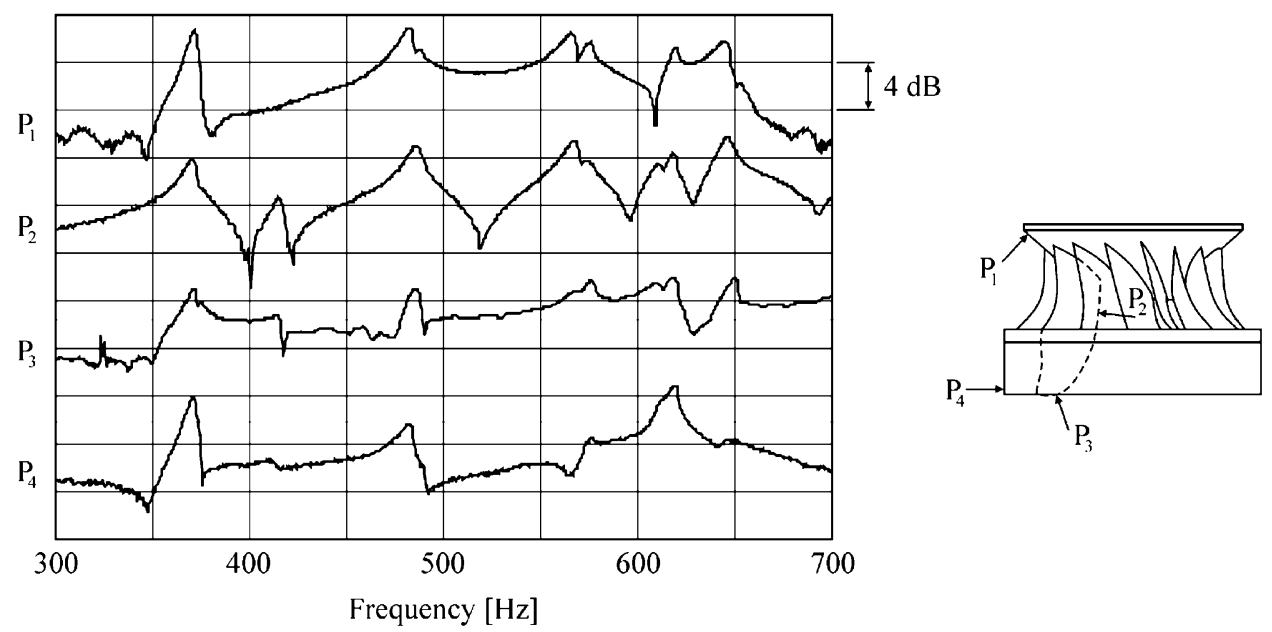

Fig. 3. FRFs between the indicated measured positions and an impact on the band.

\subsection{Testing procedure}

In modal analysis the property of reciprocity can usually be assumed. It means that the transmission between a points $p$ and $q$ is the same as between the point $q$ and the point $p$. This allows to measure in one point while impacting in several other points instead of impacting in just one point and measuring in different points. This procedure has the advantage of measuring in a few points and changing the impact position without altering the test configuration. Several preliminary tests to check the influence of the transducer and impact locations were carried out.

The impact excitation is applied to 16 positions in the band and 6 positions in each blade, giving a total of 118 impact positions. Six impact tests are done in each impact position. In these six tests, the vibratory response and impact are measured simultaneously with a duration of $2 \mathrm{~s}$, giving a frequency resolution of $0.5 \mathrm{~Hz}$.

\subsection{Effect of transducer location}

When measuring vibrations for modal analysis, it is important to avoid the location of the transducer close to any nodal point of a mode-shape. Moreover, it is also important to select the adequate impact position to excite conveniently each mode.

Some experiments are done to identify the most sensitive positions in the runner. Fig. 3 shows the runner response in air, in different positions from an impact excitation in the same position. For instance, it can be noticed that around $415 \mathrm{~Hz}$ there is a natural frequency that is not detected if the response is measured in position $\mathrm{P}_{1}$. After taking this into 
account and testing several points, the measuring and impact positions are selected. The best measuring position is located in the blades close to the band. If the vibration measurements and/or impacts are done only on the band, the modes with no deformation in the band cannot be detected. Fig. 3 is limited to the frequencies of the mode shapes studied in this paper but several more modes are present at higher frequencies. There is a difference in the shape of the peaks at the same natural frequency but measured at different positions. This is due to the phase relation between them. It does not mean that the damping ratios are different; it means that the different modes are added with different phase lag.

To determine the geometry of the modes with enough accuracy, the band and all the blades are tested. Impacts are applied on six positions in each blade and on 16 points around the band perimeter as is indicated in Fig. 4.

\subsection{Effect of tank boundaries}

The tank boundaries are analysed in order to be sure that the results are independent of the position of the runner in the tank. The proximity of a free surface reduces the added mass of a vibrating body in water and increases the damping. This occurs because an important part of the kinetic energy imparted to the fluid is used to generate surface waves dissipating it. Contrary to the effect of the free surface, the proximity of a solid boundary increases the added mass because of the build-up of pressure in its vicinity (Naudascher and Rockwell, 1994).

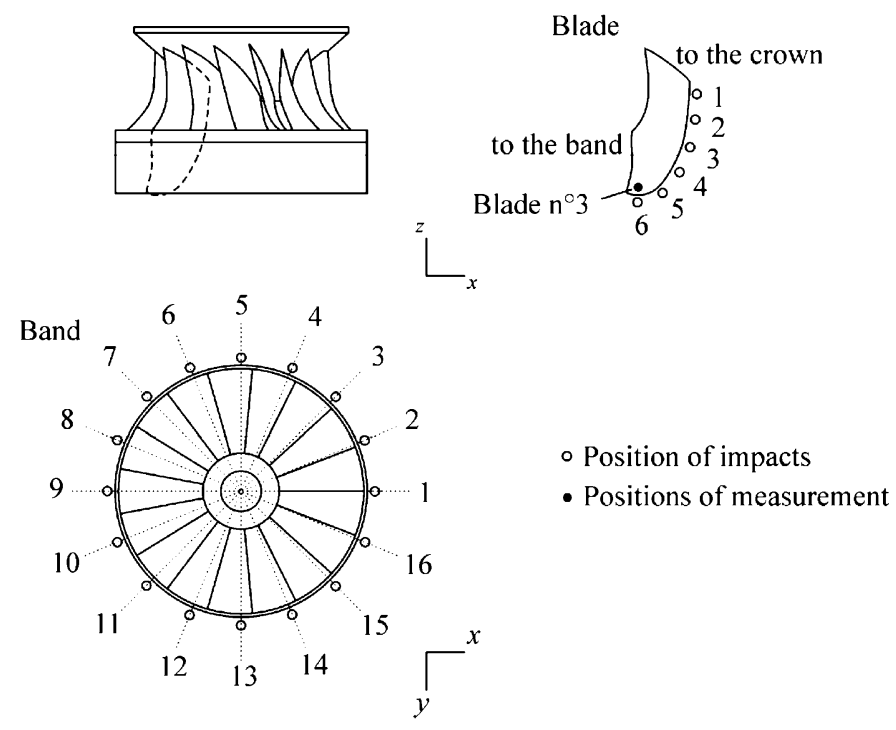

Fig. 4. Measuring and impact positions on the runner blades and band.

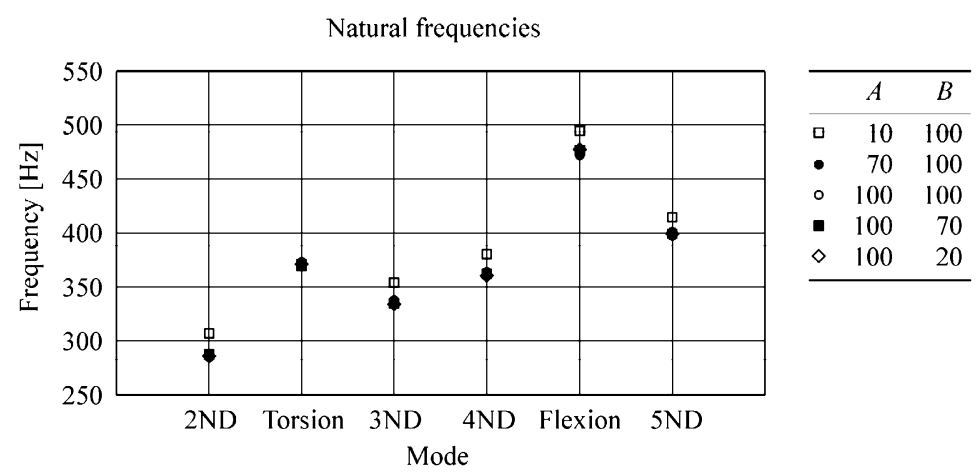

Fig. 5. Natural frequencies obtained in water for various distances to the free surface $(A)$ and to the lateral side $(B)$. 
To determine the influence of the boundaries on the system parameters, several tests are carried out with the runner submerged in water. The distances $A$, to the upper free surface, and $B$, from the runner to the lateral walls of the tank (Fig. 2), are varied and the results obtained compared.

The influence of the free surface is found to be significant when $A$ is below $0.07 \mathrm{~m}\left(A / D_{m}<0.17\right)$. For larger distances the effects are negligible. In Fig. 5, the variation in the natural frequencies for different values of $A$ and $B$ has been represented. When $A$ is $10 \mathrm{~mm}$ the change in the natural frequencies is considerable, but for the other tests with larger values it is not important. This influence also depends on the vibration mode. The influence in the system parameters of the distance from the runner to the tank wall is negligible for the values tested.

\section{Experimental results}

\subsection{Runner in air}

All the system parameters have been well determined from the experiments. Typical amplitude, phase and coherence results corresponding to an FRF obtained in one of the tests with the runner in air are shown in Fig. 6.

The main mode-shapes that are obtained with the runner in air are shown in Fig. 7. In all the plots of mode-shapes, the normalization is done considering the maximum displacement found in the whole positions of measurement. It is observed that the experimental values of the FRFs obtained with different tests remain constant. It can be seen that generally most of the mode-shapes obtained are characterised by a deformation pattern of the band. The geometry of these band modes has a clear characteristic represented in Fig. 8. At each mode, the band describes a sinusoidal displacement in the radial direction of its perimeter. This sinusoidal shape has $m$ cycles around the band perimeter with $\mathrm{m}$ being an integer. Due to that, the band has $2 m$ positions where the deformation is zero and $m$ nodal diameters (ND). To identify all these modes it is necessary to measure in the radial direction of the band in different points.

There are other modes that are not characterised by the deformation pattern of the band. In these modes, even if the band is not deformed, it performs a particular motion. These modes can be classified by this motion as torsional, flexion, and axial. In the torsional mode the band rotates with respect to the $z$-axis. In the axial mode, the band moves only in the $z$ direction, and in the flexion mode, the band moves like a pendulum. All these modes are well detected.

For a circular symmetrical structure, two repeated mode-shapes are obtained for the same natural frequency having the same shape, but with different nodal positions. In fact, this occurs because the solution of the system that represents
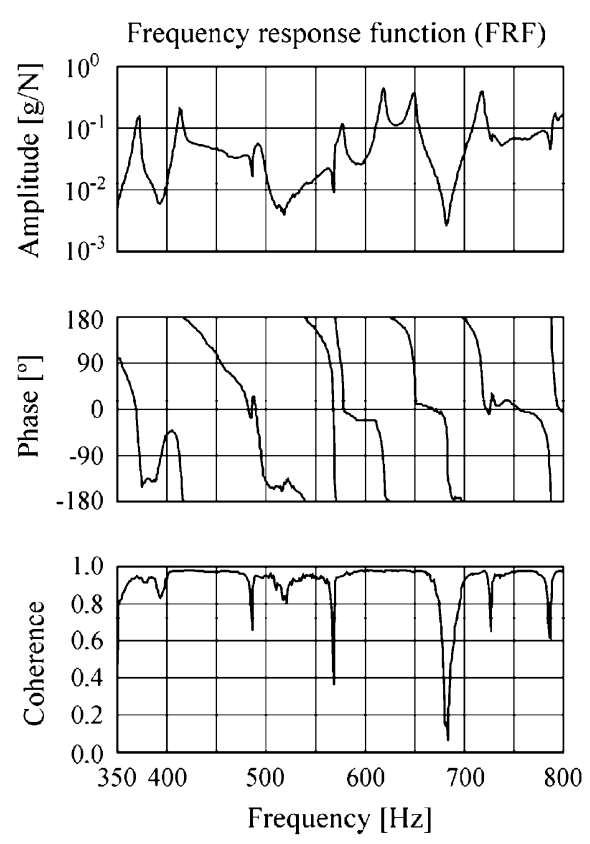

Fig. 6. Typical FRF obtained during the tests in air. 


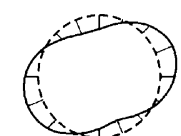

(a)

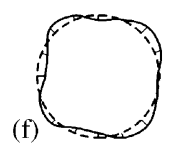

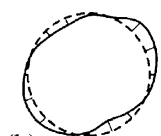

(b)

(g)

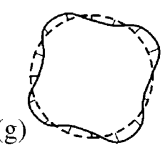

(c)

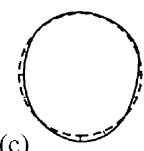

(h)

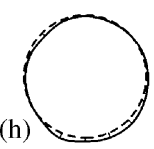

(d)

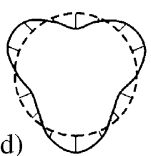

(i)

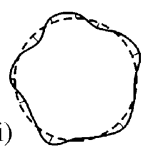

(e)<smiles></smiles>

(j)<smiles>C1CCCOCC1</smiles>

Fig. 7. Mode-shapes for the (a,b) 2ND, (c) torsion, (d,e) 3ND, (f,g) 4ND, (h) flexion and (i,j) 5ND modes in air. The corresponding natural frequencies are at 372.0, 375.0, 417.5, 484.5, 490.5, 569.5, 578.0, 616.5, 648.0 and $651.5 \mathrm{~Hz}$.

2ND
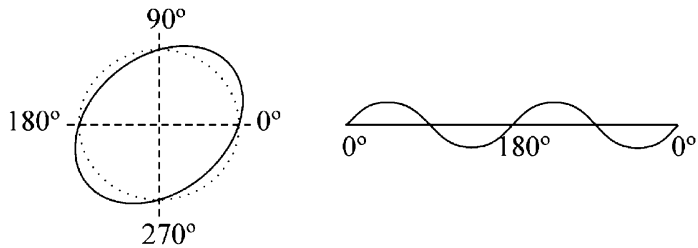

3ND
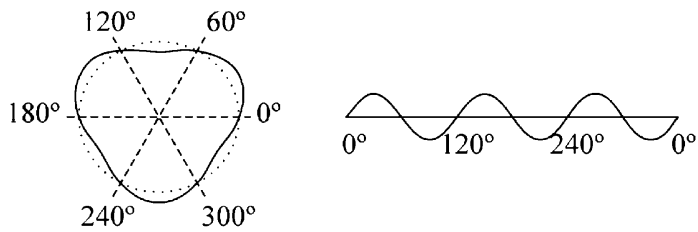

4ND
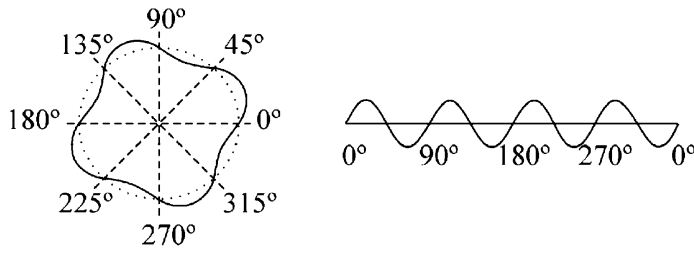

Nodal diameter

Deformation pattern

Fig. 8. Geometry of the main band modes of the runner.

\section{Band mode shape Blade mode shape}
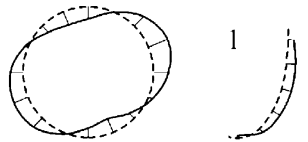<smiles>C=CC=CC</smiles><smiles>C=C=CC</smiles><smiles>CC=C=CC</smiles>

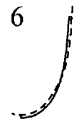

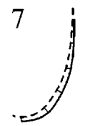<smiles>CC=C=CC</smiles><smiles>CC=C=CC</smiles><smiles>C=CCCCC</smiles>

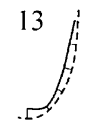

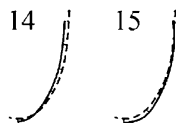

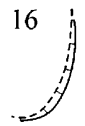<smiles>CC=CCCCC</smiles>

Fig. 9. Band and blade mode-shapes for the first 2ND band mode in air. Natural frequency at $372.0 \mathrm{~Hz}$.

a circular symmetrical structure gives two different eigenvectors (mode-shapes) for each single eigenvalue (natural frequency). These two eigenvectors are known as the sine and cosine mode-shapes. Actually, perfect symmetry is not possible; so, the two mode-shapes are obtained but not exactly at the same frequency. For instance, the first 3ND mode is found at $484.5 \mathrm{~Hz}$, and the second 3ND mode at $490.5 \mathrm{~Hz}$. In Fig. 9, the first mode-shape found at $372.0 \mathrm{~Hz}$ is 
represented. It corresponds to a two nodal diameter (2ND) band mode, which presents higher deformation in the band than in the blades. A similar deformation pattern appears in opposite blades; for instance, the mode-shape of blade 1 is comparable to the mode-shape of blade 9 .

Special attention is paid to the blade mode-shapes in order to detect and determine the modes with low deformation in the band. The torsional mode found at $417.5 \mathrm{~Hz}$ is shown in Fig. 10. The deformation of the band is low in comparison with the deformation of the blades, and as expected, all the mode-shapes in the blades are equivalent. It can be observed that the node in the blades is located in the same position. For the flexion mode, half of the blades are in phase and the other half are in opposite phase. Fig. 11 shows the axial mode which has negligible deformation in the band, it can be noticed that all the blade mode-shapes are equivalent as in the case of the torsional mode.

\subsection{Runner submerged in water}

Tests were carried out with the runner located in the centre of the water tank with a distance $A$ of $100 \mathrm{~mm}$ and with a distance $B$ of $45 \mathrm{~mm}$. The mode-shapes obtained with the submerged runner are shown in Fig. 12. The same modes than in air are obtained in water but with different frequencies and damping.

\subsection{Comparison between the response in air and in water}

In Fig. 13, the FRFs obtained in air and in water in the same position are compared indicating the corresponding modes shapes. In Table 1, the mean values of frequencies $\left(\bar{\omega}_{d}\right)$ and damping $(\bar{\zeta})$ obtained for the runner in air and submerged in water (with a subscript $W$ ), as well as their ratios, are listed. In the last columns of this table, the added mass coefficient defined in Section 1.4 is listed and can be used to extrapolate these results to runners of different material and size but with similar specific speed.

It can be noticed that the order between different types of modes does change due to the added mass. As an example, the torsion mode is after the 2ND mode in air but it is after the 4ND mode in water. However, as expected, the band modes have the same order. This means that, for instance, a $2 \mathrm{ND}$ mode always has a lower frequency than a $3 \mathrm{ND}$ mode.

The reduction in frequency due to the added mass depends on the mode-shape. It is more important in the band modes, where the reduction ratios show values from $25 \%$ in the $2 \mathrm{ND}$ mode to a $38 \%$ in the $5 \mathrm{ND}$ mode. The modes presenting low deformation in the band in comparison with the blades (torsional, flexion, and axial) are the less affected

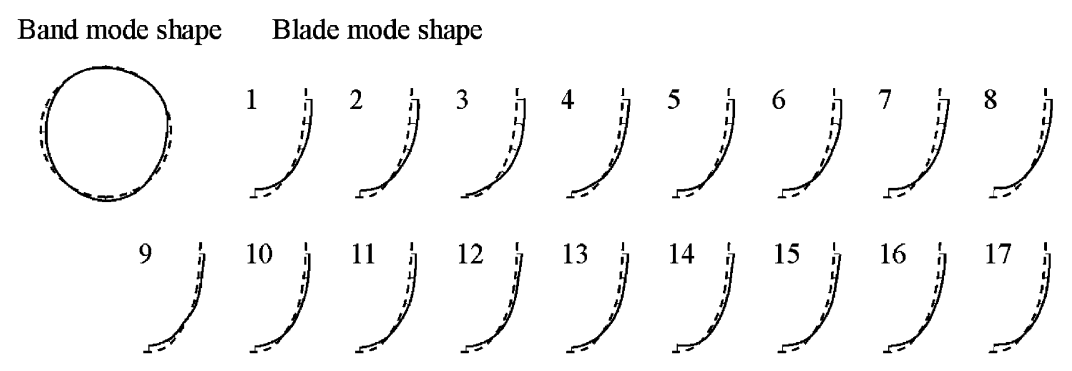

Fig. 10. Band and blade mode-shapes for the torsional mode in air. Natural frequency at $417.5 \mathrm{~Hz}$.

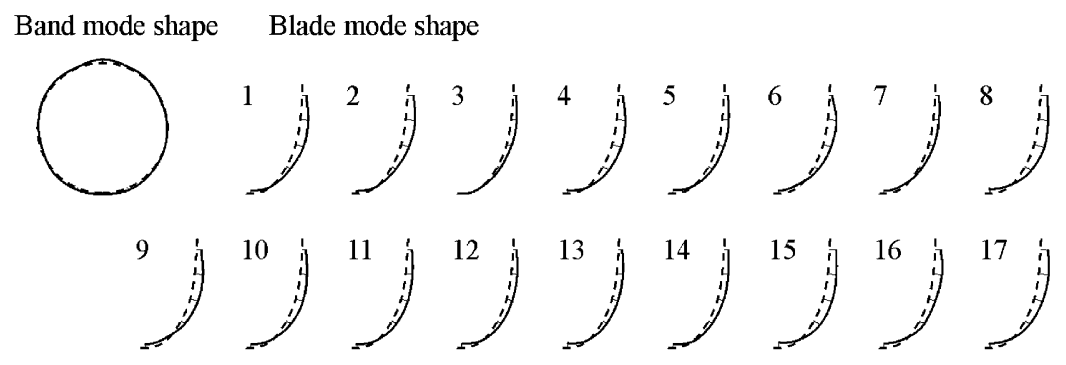

Fig. 11. Band and blade mode-shapes for the axial mode in air. Natural frequency at $869.5 \mathrm{~Hz}$. 


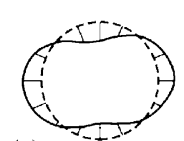

(a)

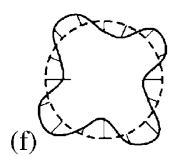<smiles></smiles>

(b)

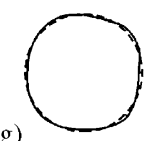

(c)

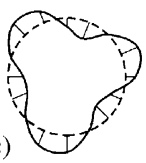

(h)

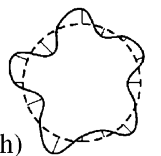

(d)

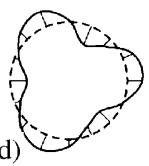

(i)

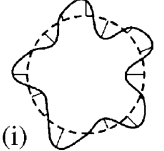

(e)<smiles>c1ccccc1</smiles>

(j)

Fig. 12. Mode-shapes for (a,b) 2ND, (c,d) 3ND, (e,f) 4ND, (g) torsion, (h,i) 5ND and (j) flexion modes in water. The corresponding natural frequencies are at 277.0, 282.0, 329.5, 333.0, 357.0, 361.0, 370.5, 397.0, 403.0 and $481.5 \mathrm{~Hz}$.

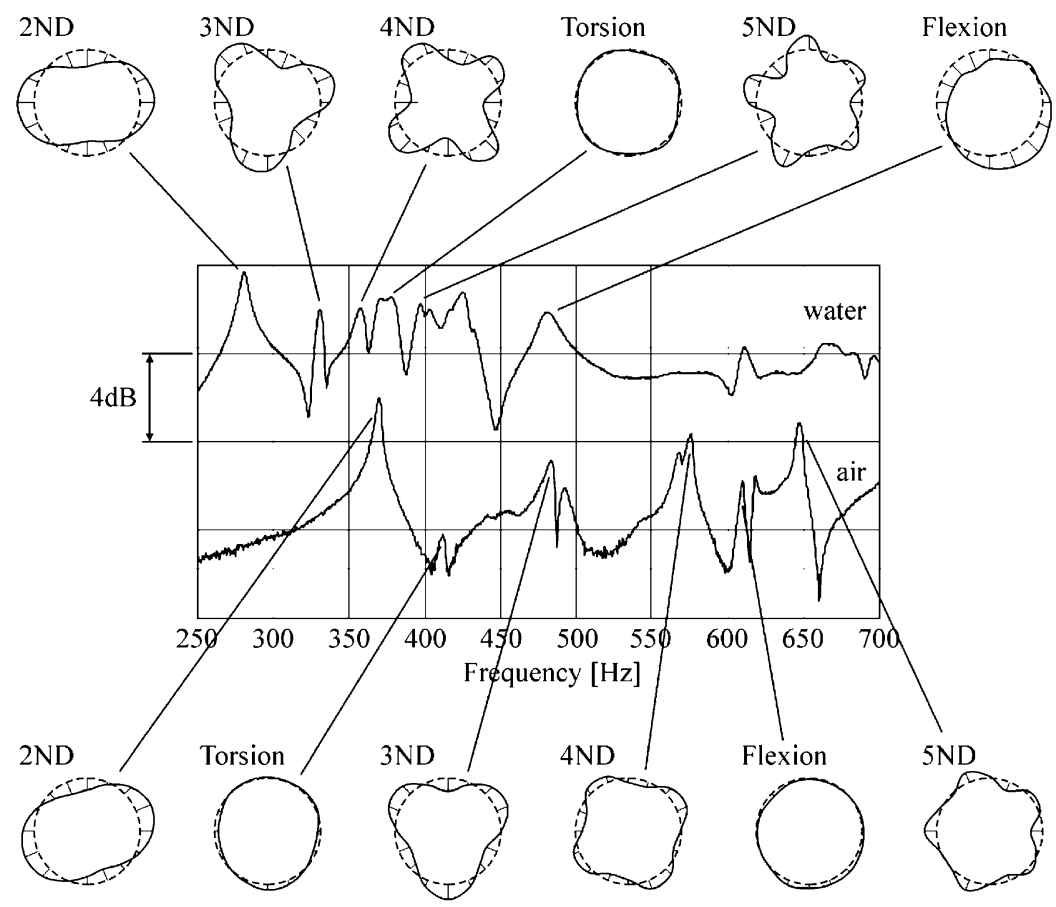

Fig. 13. Comparison between air and water modes.

Table 1

Modal analysis results for the runner in air and submerged in water

\begin{tabular}{|c|c|c|c|c|c|c|c|c|}
\hline \multirow[t]{2}{*}{ Mode } & \multicolumn{2}{|l|}{$\bar{\omega}_{d}$} & \multirow[t]{2}{*}{$\bar{\omega}_{d, W} / \bar{\omega}_{d}$} & \multicolumn{2}{|l|}{$\bar{\zeta}$} & \multirow[t]{2}{*}{$\bar{\varsigma}_{W} / \bar{\zeta}$} & \multirow[t]{2}{*}{$M_{a, i} / M_{i}$} & \multirow[t]{2}{*}{$\gamma_{i}$} \\
\hline & Air & Water & & Air & Water & & & \\
\hline $2 \mathrm{ND}$ & 373.5 & 279.5 & 0.748 & 0.0056 & 0.0070 & 1.25 & 0.79 & 6.56 \\
\hline Torsion & 417.5 & 370.5 & 0.887 & 0.0047 & 0.0068 & 1.45 & 0.27 & 2.24 \\
\hline 3ND & 487.5 & 331.3 & 0.680 & 0.0068 & 0.0082 & 1.21 & 1.16 & 9.63 \\
\hline 4ND & 573.8 & 359.0 & 0.626 & 0.0040 & 0.0070 & 1.75 & 1.55 & 12.87 \\
\hline Flexion & 616.8 & 482.5 & 0.782 & 0.0033 & 0.0120 & 3.64 & 0.64 & 5.31 \\
\hline $5 \mathrm{ND}$ & 649.5 & 400.0 & 0.616 & 0.0039 & 0.0069 & 1.77 & 1.64 & 14.69 \\
\hline Axial & 869.5 & 685.5 & 0.788 & 0.0028 & 0.0051 & 1.82 & 0.61 & 5.05 \\
\hline
\end{tabular}


by the added mass of water (for instance, $11 \%$ of reduction in frequency for the torsional mode). Because of its low band deformation in the direction of the seals, the torsional mode will not be significantly affected by the effect of nearby structures (seals). This may be caused by the fact that in band modes there is deflection in the blades and band, while in the torsional mode there is only deflection in the blades. The axial mode appears at higher frequencies $(869.5 \mathrm{~Hz}$ in air and $685.5 \mathrm{~Hz}$ in water) with a reduction ratio of $21 \%$. In the axial mode the band has no deformation. These are interesting results taking into account that many designers suppose a constant reduction of frequency for all the modes (Tanaka, 1990).

The amount of added mass in relation to the modal mass $M_{a, i} / M_{i}$ is found to be considerable. In some cases it is even higher than 1 . This high value can come from two different reasons: a high value of $M_{a, i}$; or a small value of $M_{i}$. In the case of the runner, the responsible of the high value of $M_{a, i} / M_{i}$ is the low structural mass where the thicknesses of the band and especially the blades are low. This condition can be observed in studies in simply geometries like a plate, where the thickness of the plate modifies the reduction of natural frequencies (Liang et al., 2001).

The damping found has low values in all the modes. This is the typical behaviour for a body vibrating with small amplitudes and high frequencies in stagnant fluid in the absence of wave radiation. It can be seen that the damping in water is always higher than in air. The percentage of increase obtained is at a maximum for the flexion mode.

In the last columns of the table the ratio between the modal added mass and the modal mass as well as the parameter $\gamma$ have also been included.

\section{Conclusions}

The influence of still water on the modal characteristics of a reduced scale model of a Francis turbine was experimentally investigated. In the first part of the experiment the runner was suspended in air and in the second it was suspended inside a tank full of still water. Natural frequencies and mode-shapes were determined in both cases.

An analysis to determine the influence on the results of the sensor and impact location was carried out as a preliminary task. From this analysis, the measuring and impact positions were selected. The best impact positions were in the blades close to the band. If the impacts were done only on the band the torsional mode could not be detected.

For the tests in water, the influence of the distance to the free surface and to the tank walls was determined in order to be sure that these boundaries do not affect the results. With distances from the runner to the free surface higher than $0.07 \mathrm{~m}$ the effects are found to be negligible. Dividing this value by the diameter of the runner its gives the nondimensional value $A / D_{m}=0.17$. This parameter can be taken as a reference when planning other tests.

The band, torsional, flexion, and axial modes were identified. The band mode-shapes presented several ND (2, 3, 4, $5 \ldots$ ) with two modes for each ND having almost the same frequency. In the band mode-shapes there is deformation in the band and in the blades. In the torsional, flexion and axial modes the deformation occurs only in the blades.

The effect of the added mass and damping of water is significant. The same mode-shapes obtained in air are obtained in water but with lower natural frequencies and higher damping ratios in water. The reduction in the natural frequencies depends on the mode of vibration. This reduction is at a maximum in the band modes (i.e., $25 \%$ for $2 \mathrm{ND}$ and $38 \%$ for the 5ND) and at a minimum in the torsional mode $(11 \%)$. In the band modes the reduction is related with the deformation in the band; for higher deformation, a higher reduction ratio.

The damping ratios are increased but are not high enough to affect significantly the values of the natural frequencies. Therefore, the main effect responsible for the reduction in the natural frequencies is the added mass effect.

The proximity of the seal area to the runner band can modify the results obtained for the band modes; however, other modes like the torsional and axial mode, are not affected by nearby structures.

Using nondimensional values it is possible to extrapolate the results of the particular runner tested to other runners. This allows us to predict the reduction in the natural frequencies from air to water for runners with similar geometry.

\section{Acknowledgements}

This research has been carried out as a part of the Hydrodyna Eureka project no. 3246. The authors would like to acknowledge the Voith Siemens Company (Germany) and the LMH (EPFL) of Lausanne (Switzerland) for their collaboration. Acknowledgement is also given to Alfa II project "VICONDIA". 


\section{References}

Abramson, H.N., 1969. Hydroelasticity: a review of hydrofoil flutter. ASME Applied Mechanics Review 22, $115-121$.

Albijanic, R., Marjanovic, M., Ignjatovic, B., Boskovic, V., Advic, E., 1990. Modal analysis in the dynamic identification of vital hydrounit components. In: Proceedings of 15th IAHR Symposium on Modern Technology in Hydraulic Energy Production, A3, Belgrade, Yugoslavia.

Blevins, R.D., 1995. Formulas for Natural Frequency and Mode Shape. Krieger Publishing Company, FL, USA.

Brennen, C.E., 1994. Hydrodynamics of Pumps. Concepts ETI Inc., Oxford University Press, Vermont, Oxford.

Dubas, M., Schuch, M., 1987. Static and dynamic calculation of a Francis turbine runner with some remarks in accuracy. Computers \& Structures 27, 645-655.

Cao, J.M., Chen, Ch.L., 2002. Analysis of abnormal vibration of a large Francis-turbine runner and cracking of the blades. Journal of Southwest Jiaotong University 37, 68-72.

Du, J.B., He, S.J., Wang, X.C., 1998. Dynamic analysis of hydraulic turbine runner and balde system (II) - analysis of examples. Journal of Tsinghua University (Sci \& Tech) 38, 72-75.

Egusquiza, E., Mateos, B., Escaler, X., 2002. Analysis of rotor-stator interaction in operating pump-turbines. In: Proceedings of the XXI IAHR Symposium on Hydraulic Machinery and Systems, Lausanne, Switzerland.

Ewins, D.J., 1984. Modal Testing: Theory and Practice. Research Studies Press, Hertfordshire, UK.

Fisher, R.K., Seidel, U., Grosse, G., Gfeller, W., Klinger, R., 2002. A case study in resonant hydroelastic vibration: the causes of runner cracks and the solutions implemented for the Xiaolangdi hydroelectric project. In: Proceedings of the XXI IAHR Symposium on Hydraulic Machinery and Systems, Lausanne, Switzerland.

Keim, S.R., 1956. Fluid resistance to cylinders in accelerated motion. ASCE Journal of Hydraulics Division 82 (HY6, paper 1113).

Liang, C., Liao, C., Tai, Y., Lai, W., 2001. The free vibration analysis of submerged cantilever plates. Ocean Engineering 28, 1225-1245.

Maia, N., Montalvão e Silva, J.M., 1997. Theoretical and Experimental Modal Analysis. Research Studies Press, Hertfordshire, UK.

Millán, L.D., Kazatchkov, L., De Vecchi, H.P., Fedorov, A., Beling, B., 2005. Problemas de vibraciones y fluctuaciones de presión en una turbina hidráulica de tipo Francis con amplio rango de salto. In Proceedings of Conagua, Mendoza, Spain.

Naudascher, E., Rockwell, D., 1994. Flow Induced Vibrations. Balkema, Rotterdam.

Sarpkaya, T., 1975. Forces on cylinders and spheres in a sinusoidally oscillating fluid. ASME Paper 75-APMW-27, Applied Mechanics Western Conference, Honolulu, USA.

Tanaka, H., 1990. Vibration behaviour and dynamic stresses of runners of very high head reversible pump-turbines. In: Proceedings of 15th IAHR Symposium on Modern Technology in Hydraulic Energy Production, U2, Belgrade, Yugoslavia.

Xiao, R.F., Wei, C.X., Han, F.Q., Zhang, S.Q., 2001. Study on dynamic analysis of the Francis turbine runner. Journal of Large Electric Machine and Hydraulic Turbine 7, 41-43. 